\title{
An Update on Role of Matrix Metalloproteinases in Oral Health
}

\section{Adithya R Pillai*, K Revathi and N Arunagirinathan}

Central Research Laboratory, Meenakshi Academy of Higher Education and Research, Chennai, India

*Corresponding Author: Adithya R Pillai, Central Research Laboratory, Meenakshi Academy of Higher Education and Research, Chennai, India.

Received: August 27, 2019; Published: September 05, 2019

DOI: $10.31080 /$ ASPS.2019.03.0390

\begin{abstract}
MMPs (matrix metalloproteinases) are involved in physiological and pathological conditions in the human body. In the oral health, MMPs have been manifested in certain oral diseases. Increased secretion of MMPs has been reported in chronic inflammatory conditions, including periodontitis, and leads to irreversible soft tissue destruction. As excessive production of MMPs is associated with several pathological processes, including cancer metastasis and chronic inflammatory conditions, over the last 20 years the synthesis of a variety of low-molecular weight MMP inhibitors has occurred, even though the majority are not commercially available. This review is focusing on the role of matrix metaloproteinases in oral diseases to date. This review will be really a guide for the researchers and dentist who are interested to know about MMPs in different oral health.
\end{abstract}

Keywords: MMPs; Oral Health; Periodontitis

\section{Introduction}

Matrix metallo proteinases are a group of enzymes which is calcium dependent zinc containing endopeptidases. There groups are collagenase, gelatinases, stromelysins, membrane-type MMPs [1]. There are namely MMP1 to MMP 28 different types are identified. The MMPs play an important role in tissue remodeling associated with various physiological or pathological processes such as morphogenesis, angiogenesis, tissue repair, cirrhosis, arthritis, and metastasis. MMP-2 and MMP-9 are thought to be important in metastasis. MMP-1 is thought to be important in rheumatoid arthritis and osteoarthritis. Recent data suggests active role of MMPs in the pathogenesis of Aortic Aneurysm. Excess MMPs degrade the structural proteins of the aortic wall. Disregulation of the balance between MMPs and TIMPs is also a characteristic of acute and chronic cardiovascular diseases [2]. All MMPs are synthesized in the latent form (Zymogen). They are secreted as proenzymes and require extracellular activation. They can be activated in vitro by many mechanisms including cleaving of peptide, chaotropic agents, and other proteases. The MMPs are inhibited by specific endogenous tissue inhibitor of metalloproteinases (TIMPs), which comprise a family of four protease inhibitors: TIMP-1, TIMP-2, TIMP-3, and TIMP-4. Synthetic inhibitors generally contain a chelating group that binds the catalytic zinc atom at the MMP active site tightly [3]. Common chelating groups include hydroxamates, carboxylates, thiols, and phosphinyls. Hydroxymates are particularly potent inhibitors of
MMPs and other zinc-dependent enzymes, due to their bidentate chelation of the zinc atom. Other substituents of these inhibitors are usually designed to interact with various binding pockets on the MMP of interest, making the inhibitor more or less specific for given MMPs [3].

MMPs in periodontitis

Periodontitis is a bacterial infection which induce the inflammation of the periodontal cavity in humans. And if left untreated it can lead to serious issues of bone loss. 50-60\% of adult population of our country has chronic periodontitis. This disease is having more susceptibility to diabetes and other cardio vascular diseases [4]. There are researches showing the mechanism behind the periodontitis. As it is a bacterial infection, polymicrobial colonization have been shown by the researchers. Both aerobic and anaerobic bacteria have been identified. The major concern of all periodontist is the gradual bone degradation in the chronic periodontitis [5]. And then the role of MMPs have been studied to understand the actual cause of bone degradation. There are research showing the role of MMP8, MMP9, MMP2 in the periodontitis.

MMP-8 and MMP-9 are the most abundant MMPs in periodontal tissues reflecting periodontal disease severity, progression, and treatment response [6]. They are secreted by infiltrating polymorphonuclear leukocytes, but also macrophages, plasma cells, and resident cells - such as fibroblasts, endothelial cells, keratinocytes 
and bone cells. MMP-13 is a collagenase that has been detected in fibroblasts, macrophages, osteoblasts, plasma cells, and gingival epithelial cells. Though less abundant, MMP-13 has been implicated in periodontal soft tissue destruction and, along with MMP-9, it has been involved in alveolar bone resorption and periodontal tissue breakdown. The mechanisms regulating MMP activation may vary depending on the specific tissue and disease microenvironment. During periodontitis progression, these MMPs can be activated by independent or co-operative cascades involving pathogen and host proteases.

MMP proteolytic cascades can lead to widespread periodontal tissue destruction due to cooperative MMP activation and might represent an interesting target for diagnostics and therapy. MMP13 is able to induce proMMP-9 activation and MMP-13 auto-activation by self-proteolysis in vitro A previous study from our group reported that MMP-13 induces proMMP- 9 activation in gingival tissues from periodontitis patients [7]. MMP-9, in turn, can activate proMMP-2 and proMMP-13 in vitro. Collagenase levels of MMP-14, MMP-8, and MMP-13, have been correlated in chronic periodontitis patients, which suggests either a co-operative role and/or the potential settlement of collagenase activation cascades [8]. MMP14 can activate MMP-8 and - 13 in vitro, as well as MMP-2, and has been correlated in vivo with active MMP-13 in periodontitis sites. Altogether, these mechanisms could enhance periodontal tissue breakdown and consequent chronic periodontitis progression [8].

In periodontitis, matrix metalloproteinase- 8 is the main collagenase in gingival connective tissue, Also, $90-95 \%$ of collagenolytic activity in gingival crevicular fluid originates from matrix metalloproteinase-8 Overall, the increased gingival crevicular fluid collagenase activity and matrix metalloproteinase- 8 levels have been found to correlate with the levels of type I collagen degradation products, overcoming the protective shield provided by tissue inhibitors of matrix metalloproteinase- 1 in disease-active/untreated sites compared with inactive sites from periodontitis patients and healthy subjects. Successful periodontal treatment and adjunctive matrix metalloproteinase-inhibitory medication have been shown to cease the progression of periodontitis and reduce the levels of matrix metalloproteinases in oral fluids. Conventional hygienephase periodontal treatment decreases the gingival crevicular fluid matrix metalloproteinase-8 levels (but in gingival crevicular fluid from patients with periodontitis, the decreased matrix metalloproteinase-8 levels often remain at a higher level compared with those found in periodontally healthy subjects [9].

\section{Smoking and matrix metalloproteinase}

Smoking is a well-known behavioral risk factor for periodontitis with influence on the host inflammatory response. For this reason it can be assumed that smoking has an effect on the oral matrix metalloproteinase- 8 response that should be considered when matrix metalloproteinase-8 levels of oral fluid samples are used as a diagnostic aid. Neutrophil granulocytes are the main source of oral matrix metalloproteinase-8 but many other cell types, including periodontium resident cells, can release matrix metalloproteinase- 8 when triggered by microbes [10].

The matrix metalloproteinase levels in the systemic circulation of smokers are elevated or decreased compared with those in the systemic circulation of non -smokers. However, the serum matrix metalloproteinase-8 levels of systemically healthy smokers and nonsmokers are similar. On the other hand, the serum levels of matrix metalloproteinase- 8 and tissue inhibitors of matrix metalloproteinase- 1 in periodontitis patients who smoke are lower than those of nonsmokers, giving an insight to the disturbed matrix metalloproteinase-8/tissue inhibitors of matrix metalloproteinase-1 ratio in the blood serum of smokers. In a study analyzing expression of matrix metalloproteinase-8 mRNA, induced by smoking, in peripheral blood samples, Morozumi., et al. observed that smokers had lower levels of matrix metalloproteinase-8 mRNA compared with nonsmokers, but the levels increased significantly 8 weeks after cessation of smoking [11].

Liu., et al. detected stronger matrix metalloproteinase-8 expression in the gingival connective tissue of patients with chronic periodontitis who smoke, compared with the gingival connective tissue of nonsmokers. They concluded that smoking might induce a local increase of matrix metalloproteinase- 8 burden with risk of periodontitis progression. In a treatment study observing the effect of periodontal surgery on matrix metalloproteinase-8 levels of gingival crevicular fluid in smokers and nonsmokers, the matrix metalloproteinase-8 levels in gingival crevicular fluid of smokers were similar before and after surgery; however, in nonsmokers, the matrix metalloproteinase-8 levels of gingival crevicular fluid decreased during the recovery period following surgical treatment, indicating that matrix metalloproteinase- 8 may play a role in the inferior treatment response of smokers [12].

\section{MMPs in oral rinse}

Mouth rinse or oral-rinse samples have been shown to reflect total gingival crevicular fluid matrix metalloproteinase-8 activity, giving a general view of the oral inflammatory status. Mouth rinse matrix metalloproteinase-8 levels have revealed good correlations with the oral inflammatory burden, measured using clinical periodontal and health parameters, when matrix metalloproteinase-8 detection methods using the same matrix metalloproteinase- 8 antibodies (dento ELISA and IFMA) were applied but not with the traditional ELISA method [12].

Recently, a point-of-care/chair-side matrix metalloproteinase-8 immunoassay, which utilizes a lateral flow immunoassay and the same monoclonal antibody as previously mentioned assays (dipstick, IFMA and dentoELISA) was developed to detect and measure mouthrinse levels of matrix metalloproteinase-8. Nwhator, et al. recruited 76 men, requiring seminal fluid analysis as part of another study, to investigate the correlations between this novel lat- 
eral-flow matrix metalloproteinase-8 mouthrinse point-of-care/ chair-side Oral Risk Indicator immunoassay and clinical periodontal parameters (bleeding on probing, oral hygiene and periodontal pockets). Basic dental and periodontal examinations were carried out, in which pocket depths were measured using a Community Periodontal Index of Treatment Needs probe, and debris/plaque and calculus indices were recorded. Participants were subsequently grouped into 'good,' 'fair' and 'poor' oral-hygiene [13].

In a qualitative analysis of matrix metalloproteinase- 8 levels among pregnant women, 117 (87.3\%) of 134 were found to have elevated matrix metalloproteinase-8 levels in mouthrinse. It was reasoned that there appears to be an unexplained racial difference in matrix metalloproteinase-8 levels among Black pregnant women compared with their White counterparts [13]. Should this be confirmed from larger studies, it might explain racial differences in preterm labor.

\section{Conclusion}

Overall, matrix metalloproteinases is a promising candidate for oral fluid (gingival crevicular fluid, mouthrinse and saliva) chairside/point-of-care diagnostics, for predicting, diagnosing and determining the progressive phases of the episodic periodontitis and peri-implantitis, as well as for monitoring treatments and medications. Salivary matrix metalloproteinase-8, when used together with interleukin-1beta and $P$. gingivalis to calculate cumulativerisk score at the subject level, can be a successful diagnostic tool, especially in large-scale public health surveys, in which a thorough periodontal examination is not feasible. A high concentration of serum matrix metalloproteinase-8 was associated with subclinical atherosclerosis, as judged by carotid artery intima-media thickness, prevalent cardiovascular disease and acute coronary syndrome [14]. In cardiovascular disease-free subjects at baseline, a high level of serum matrix metalloproteinase- 8 is associated with incident acute myocardial infarction and other cardiovascular disease events, as well as death from coronary heart disease, cardiovascular disease or any cause. Also, the regulators of matrix metalloproteinase-8 activity, serum tissue inhibitors of matrix metalloproteinase- 1 and myeloperoxidase, may play important roles in the manifestation and recurrence of atherosclerotic disease [14].

\section{Acknowledgments}

The work was supported, in part, by the Meenakshi Academy of Higher Education and Research, Chennai -600078 and central research laboratory, Meenakshi ammal dental college, Chennai 600095.

\section{Bibliography}

1. Tuomainen AM., et al. "Serum tissue-degrading proteinases and incident cardiovascular disease events". European Journal of Preventive Cardiology 21 (2014): 806-812.
2. Tuomainen AM., et al. "Serum matrix metalloproteinase-8 concentrations are associated with cardiovascular outcome in men". Arteriosclerosis, Thrombosis, and Vascular Biology 27 (2007): 2722-2728.

3. Pussinen PJ., et al. "The balance of serum matrix metalloproteinase- 8 and its tissue inhibitor in acute coronary syndrome and its recurrence". International Journal of Cardiology 167 (2013): 362-368.

4. Nwhator SO., et al. "Black women's predisposition to preterm birth; could we be near the answer?" International Journal of Tropical Disease and Health 4 (2014): 194-203.

5. Nwhator SO., et al. "Clinical correlates of a lateral-flow immunoassay oral risk indicator". Journal of Periodontology (2014): 85.

6. Ozcaka 0., et al. "Smoking and matrix metalloproteinases, neutrophil elastase and myeloperoxidase in chronic periodontitis". Oral Disease 1 (2011): 68-76.

7. Gangbar S., et al. "Identification of polymorphonuclear leukocyte collagenase and gelatinase activities in mouthrinse samples: correlation with periodontal disease activity in adult and juvenile periodontitis". Journal of Periodontal Research 25 (1990): 257-267.

8. Leppilahti JM., et al. "Oral rinse matrix € metalloproteinase-8 point-of-care immuno test identifies patients with strong periodontal inflammatory burden". Oral Disease 17 (2011): 115-122.

9. Persson L., et al. "Effect of tobacco $€$ smoking on neutrophil activity following periodontal surgery". Journal of Periodontology 74 (2003): 1475-1482.

10. Liu KZ., et al. "Increased local matrix metalloproteinase-8 expression in the periodontal connective tissues of smokers with periodontal disease". Biochimica et Biophysica Acta 1762 (2006): 775.

11. Hernandez Rios M., et al. "Proteolytic roles of matrix metalloproteinase (MMP)-13 during progression of chronic periodontitis: Initial evidence for MMP-13/MMP-9 activation cascade". Journal of Clinical Periodontology 36 (2009): 1011-1017.

12. Leppilahti JM., et al. "Matrix metalloproteinases and myeloperoxidase in gingival crevicular fluid provide site-specific diagnostic value for chronic periodontitis". Journal of Clinical Periodontology 41 (2014): 348-356. 
13. Heikkinen AM., et al. "Smoking affects diagnostic salivary periodontal disease biomarker levels in adolescents". Journal of Periodontology 81 (2010): 1299-1307.

14. Mantyla P., et al. "Monitoring periodontal disease status in smokers and nonsmokers using a gingival crevicular fluid matrix metalloproteinase-8-specific chair-side test". Journal of Periodontal Research 41 (2006): 503-512.

\section{Volume 3 Issue 10 October 2019}

(C) All rights are reserved by Adithya R Pillai., et al. 